\title{
Ellipse Detection with Elemental Subsets
}

\author{
Peter Veelaert \\ University College Ghent, Engineering Sciences - Ghent University Association, \\ Schoonmeersstraat 52, B9000 Ghent, Belgium \\ peter.veelaertahogent.be
}

\begin{abstract}
We propose a simple method for fitting ellipses to data sets. The method first computes the fitting cost of small samples, called elemental subsets. We then prove that the global fitting cost can be easily derived from the fitting cost of the samples. Since fitting costs are computed from small samples, the technique can be incorporated in many ellipse detection and recognition algorithms, and in particular, in algorithms that make use of incremental fitting. Some of the theoretical results are formulated in the more general setting of implicit curve fitting.
\end{abstract}

\section{Introduction}

Due to the ubiquitous presence of round objects in natural scenes and the need for robust recognition algorithms, ellipse detection and fitting remains an important research problem in image processing as well as metrology. Many recently proposed detection algorithms are multistage processes that combine several techniques. Zhang and Liu developed a method for robust, real-time ellipse detection [8]. They propose a global multistage algorithm paying attention to several important issues in shape recognition: robust edge detection, removal of spurious points, an efficient implementation of a realtime ellipse Hough transform, and proven accuracy. A somewhat different approach is followed in a recent paper by Qiao and Ong. They use the concepts of model-based distance and angular connectivity in an iterative algorithm for ellipse fitting [6].

Also in the field of digital geometry ellipses and conics have received some attention. Coeurjolly et al have proposed a circle recognition algorithm which constructs the polygon in which the center of the circle must lie, and verifies whether this polygon is empty or not [1]. Earlier, some work had been done on the coding of conic sections by Zunic and Sladoje [9]. These few, isolated results stand in stark contrast to the amount of work done in this field on the recognition of digital straight lines and digital planes.

In this paper we focus on one particular aspect of ellipse detection: the selection of small subsets, called elemental subsets, from an edge map to estimate the ellipse parameters. The results are useful in several ways. When developing new algorithms for real-time ellipse detection (as in [8]), incremental tracking (as in [6]) or fitting (as in [7]), a better understanding of the quality of the point samples will facilitate and improve the detection process. In fact, the concept of elemental subsets has already proved to be useful for developing fast, incremental fitting algorithms for the segmentation of edge maps into lines and parabola [4, 5, 2]. In this work we provide the theoretical framework for incremental fitting with ellipses. 
Although elemental subsets have been introduced in previous work [4], in this paper we introduce signed elemental subsets and the related concept of elemental thickness. Furthermore we examine the relation between elemental thickness and algebraic thickness. The focus is on ellipse recognition, but some of the theoretical results are formulated in a more general setting: fitting implicit functions to discrete point sets.

\section{Algebraic and Elemental Thickness}

\subsection{Algebraic Thickness}

Suppose we are given a finite set $S \subset \mathbb{Z}^{2}$, and a family of curves $C$. How can we determine whether there is a curve in $C$ that will pass "close" to all the points of $S$ ? The answer to this question depends in a crucial way on the definition of closeness, a notion which can be expressed in various ways. For example, for digital straight lines the distance between a line $y=a x+b$ and a grid point $p_{i}=\left(x_{i}, y_{i}\right)$ is often defined as $\left|y_{i}-a x_{i}-b\right|$. It is the Euclidean distance measured along a vertical line crossing the point $p_{i}$. Alternatively, closeness could also be measured as $\left|\left(y_{i}-a x_{i}-b\right) / a\right|$, which coincides with the shortest Euclidean distance between the point $\left(x_{i}, y_{i}\right)$ and the line, but with the drawback that the parameter $a$ now occurs in a non-linear way.

A notion related to closeness is the concept of supporting curves. Suppose we have found a line $y=a x+b$ such that all points of $S$ satisfy $\left|y_{i}-a x_{i}-b\right| \leq \epsilon$, where $\epsilon$ has been chosen as small as possible. Then all the points lie between the straight lines $y-a x-b=\epsilon$ and $y-a x-b=-\epsilon$. These two parallel lines encompass (or support) the set $S$ as tightly as possible.

In this work we shall use the algebraic distance to measure how close a set of points lies to a curve. Let $C$ be a family of curves implicitly defined as $f\left(x, y ; a_{1}, \ldots, a_{n}\right)=0$, where $f$ is a real polynomial. The $n$ parameters $a_{1}, \ldots, a_{n}$ parametrize the family. The algebraic distance between the point $\left(x_{i}, y_{i}\right)$ and the curve is simply defined as

$$
f\left(x_{i}, y_{i} ; a_{1}, \ldots, a_{n}\right) .
$$

Although the algebraic distance differs from the Euclidean distance, in particular where the curvature of a curve is high, the algebraic distance has the advantage of simplicity. Furthermore, our aim is to find approximating curves that pass at a given distance between points, which can be accomplished relatively easily with algebraic distances.

Definition 1. Let $S$ be a finite subset of $\mathbb{Z}^{2}$ and let $C$ a family of curves defined by $f\left(x, y ; a_{1}, \ldots, a_{n}\right)=0$, where $f$ is a real polynomial in $x, y$ and the parameters $a_{1}, \ldots, a_{n}$. The algebraic thickness $\tau_{a}$ of $S$ is defined as

$$
\tau_{a}(S)=\min _{a_{1}, \ldots, a_{n}}\left(\max _{(x, y) \in S}\left|f\left(x, y ; a_{1}, \ldots, a_{n}\right)\right|\right) .
$$

Thus the algebraic thickness $\tau_{a}(S)$ is the smallest real number for which there exists a curve $f\left(x, y ; a_{1}, \ldots, a_{n}\right)=0$ in $C$ such that all the points of $S$ lie within algebraic distance $\tau_{a}(S)$ of the curve. The curve for which the minimum is achieved is called the algebraic best fit. 


\subsection{Elemental Thickness and Supporting Systems}

Supporting lines are known from digital straight lines. Suppose we have a set $S$ of grid points and a line $y=a x+b$ such that all the points of $S$ satisfy $\left|y_{i}-a x_{i}-b\right| \leq \tau_{a}(S)$, where $\tau_{a}(S)$ denotes the algebraic thickness of $S$ as defined for the family of straight lines. Then it is a well-known property that there are at least three points that lie on the two supporting lines $y_{i}-a x_{i}-b= \pm \tau_{a}(S)$ [4]. Moreover, $\tau_{a}(S)$ can be found by examining all 3-point subsets of $S$ and computing the thickness for each of them separately. The thickness $\tau_{a}(S)$ is simply the maximum of all the 3-point thicknesses. Thus elemental subsets provide a powerful tool for finding the best algebraic linear fit to a set of points. It suffices to compute the algebraic thickness of all 3-points subsets. The subset with the largest thickness yields the supporting lines and the best fit for the entire set. Because of its proven usefulness for lines we will extend the concept of supporting curves to implicitly defined curves of general type.

Definition 2. Let $C$ a family of curves be defined by $n$ parameters $a_{i}$, and let $S$ be a finite subset of $\mathbb{Z}^{2}$ containing at least $n+1$ points. An elemental subset of $S$ is a subset of $n+1$ distinct points of $S$. A signed elemental subset is an elemental subset $S$ where either the sign + or - has been assigned to each point.

For example, $\left\{p_{1}^{+}, p_{2}^{+}, p_{3}^{-}, p_{4}^{-}, p_{5}^{+}\right\}$could be a signed elemental subset for a family parametrized with 4 parameters. The aim of the signs is to determine at which side of a curve the points lie. In this case the points $p_{1}, p_{2}, p_{5}$ all lie on the same side while $p_{3}, p_{4}$ lie on the opposite side. We also introduce a sign vector $\sigma$, which in this example is equal to $\sigma=\left(\sigma_{i}\right)=(+1,+1,-1,-1,+1)$.

Definition 3. Let $C$ a family of curves be defined by $n$ parameters. A supporting system $\left\{U_{\sigma}, C^{0}, C^{+\epsilon}, C^{-\epsilon}\right\}$ consists of the following parts:

- a signed elemental subset $U_{\sigma}$;

- a real curve $C^{0}$ of the form $f\left(x, y, a_{1}, a_{2}, \ldots\right)=0$ in $C$ and a value for $\epsilon$ such that the points $p_{i}=\left(x_{i}, y_{i}\right)$ of $U_{\sigma}$ satisfy the $n+1$ equations

$$
f\left(x_{i}, y_{i}, a_{1}, a_{2}, \ldots\right)=\sigma_{i} \epsilon, \text { for } i=1, \ldots, n+1 .
$$

- the two real curves $C^{+\epsilon}$ and $C^{-\epsilon}$ defined by $f\left(x, y, a_{1}, a_{2}, \ldots\right)=\epsilon$ and $f\left(x, y, a_{1}, a_{2}, \ldots\right)=-\epsilon$, respectively.

If such a system exists, the value for $\epsilon$ is called the elemental thickness of the system, and it will be denoted as $\tau_{e}\left(\left\{U_{\sigma}, C^{0}, C^{+\epsilon}, C^{-\epsilon}\right\}\right)$. The two curves $C^{+\epsilon}$ and $C^{-\epsilon}$ are called supporting curves for $U_{\sigma}$. The curve $C^{0}$ is called an elemental best fit for $U_{\sigma}$. The sign $\sigma_{i}$ determines at which side the point $\left(x_{i}, y_{i}\right)$ lies from $C^{0}$.

Definition 3 actually gives us a means for computing supporting systems for an elemental subset. Given the signs $\sigma_{i}$ it is sufficient to solve (2), for the unknowns $a_{j}$ and $\epsilon$. Supporting systems can therefore be computed for a wide variety of curve families. Furthermore, according to Definition 3 the elemental best fit always belongs to the family of curves $C$. For the two supporting curves $C^{+\epsilon}$ and $C^{-\epsilon}$, however, this may not be true, depending on the way in which the family of curves has been defined. 
Example. We consider the family of lemniscates with equations of the form

$$
-2 a^{2}\left((-p+x)^{2}-(-q+y)^{2}\right)+\left((-p+x)^{2}+(-q+y)^{2}\right)^{2}=0 .
$$

As shown in Fig. 1, the signed elemental subset $\left\{p_{1}=(34,14)^{-}, p_{2}=(38,15)^{+}\right.$, $\left.p_{3}=(29,8)^{-}, p_{4}=(18,17)^{+}\right\}$has two supporting curves. The inner curve is not connected and consists of two separate components. The best fit is a lemniscate (the figure eight), which passes between the points of the signed elemental subset. The best fit was computed by solving the system $-2 a^{2}\left((-p+x)^{2}-(-q+y)^{2}\right)+\left((-p+x)^{2}+\right.$ $\left.(-q+y)^{2}\right)^{2}=\sigma_{i} \epsilon, i=1, \ldots, 4$, with $\sigma_{i}=-1,1,-1,1$, for $a, p, q$ and $\epsilon$. In this family the supporting curves are not lemniscates themselves, but their points lie at a constant algebraic distance $(\epsilon$ or $-\epsilon)$ of a lemniscate.

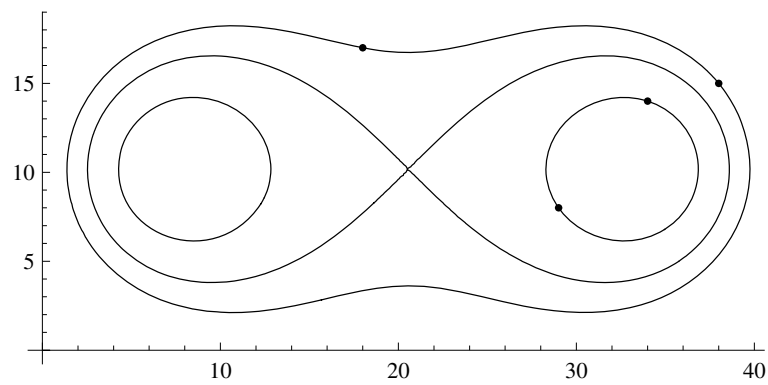

Fig. 1. A supporting system for lemniscates

From the elemental thicknesses of the supporting systems we derive the elemental thickness of an elemental subset.

Definition 4. Let $U$ be an elemental subset with $n+1$ points and let $C$ be a family curves defined by $n$ parameters. The elemental thickness of $U$ is defined as $\tau_{e}(U)=$ $\min _{\sigma} \tau_{e}\left(\left\{U_{\sigma}, C^{0}, C^{+\epsilon}, C^{-\epsilon}\right\}\right)$, where the minimum is taken over all the $2^{n+1}$ possible ways in which signs can be assigned to the points of $U$ and over all the supporting systems of each $U_{\sigma}$.

Fig. 2 shows another supporting system for the same points as in Fig. 11 Three points lie on $C^{-\epsilon}$ and one point on $C^{+\epsilon}$. The supporting curves enclose the set $U$ much more tightly than in Fig.1. In fact, there is no other supporting system with smaller thickness, which means that the $\epsilon$ of the supporting system in Fig. 2 is also the elemental thickness of $U$ as defined in Definition 4

\subsection{Relation between Algebraic and Elemental Thickness}

Up to now we have introduced two distinct forms of thickness. Algebraic thickness is defined by an optimization problem, while elemental thickness can be found by solving systems of equations. Both concepts will coincide, however, if we impose additional constraints on how a family of curves is parametrized. While all the previous definitions hold even when some of the parameters $a_{i}$ occur in a non-linear way, in the 


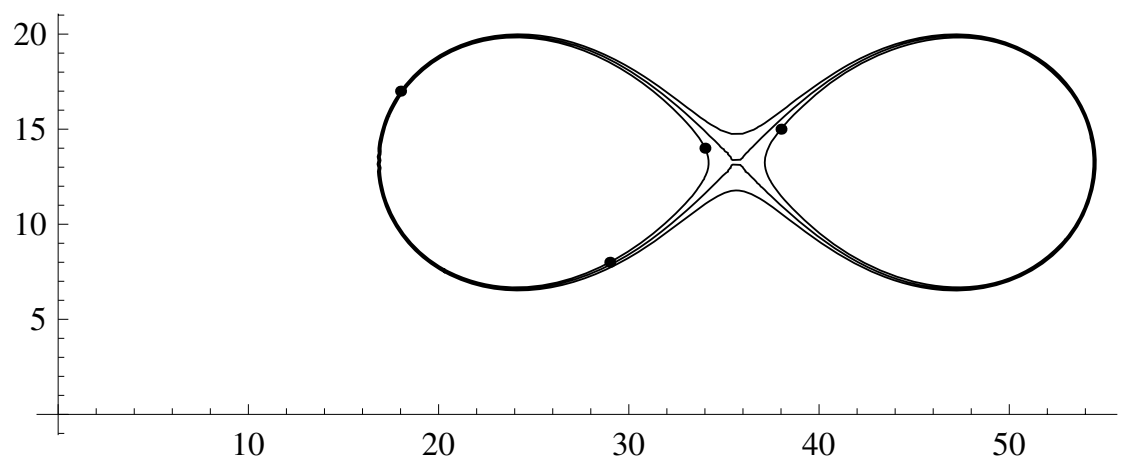

Fig. 2. The supporting system for lemniscates, which yields the minimum elemental thickness

next theorem, we add the assumption that the family $C$ consists of curves of the form $a_{1} g_{1}(x, y)+\cdots+a_{n} g_{n}(x, y)=1$, where the $g_{i}$ are polynomials, and the parameters $a_{j}$ must all occur in a linear way.

Theorem 1. Let $U$ be an elemental subset, and let $C$ be the family of curves defined as $a_{1} g_{1}(x, y)+\cdots+a_{n} g_{n}(x, y)=1$, where the $g_{i}$ represent $n$ real polynomials. Then the elemental thickness of $U$ is equal to the algebraic thickness of $U$.

Proof. Consider the following linear programming problem:

Minimize the objective function $\epsilon$ subject to the $2 n+2$ constraints

$$
\begin{aligned}
a_{1} g_{1}\left(x_{i}, y_{i}\right)+\cdots+a_{n} g_{n}\left(x_{i}, y_{i}\right)-1 \leq \epsilon, & i=1, \ldots, n+1 \\
\epsilon \leq a_{1} g_{1}\left(x_{i}, y_{i}\right)+\cdots+a_{n} g_{n}\left(x_{i}, y_{i}\right)-1, & i=1, \ldots, n+1 .
\end{aligned}
$$

This minimal value for $\epsilon$ is equal to the algebraic thickness of the elemental subset $U$. In fact, the above linear programming problem clarifies how the algebraic thickness $\tau_{a}(U)$ of Definition 1 can be computed for families with linear parameters.

Furthermore, since the $2 n+2$ inequalities define a polyhedron while the objective function is a linear function, the minimum will be attained in at least one of the vertices of the polyhedron. This vertex satisfies equations of the form

$$
a_{1} g_{1}\left(x_{i}, y_{i}\right)+\cdots+a_{n} g_{n}\left(x_{i}, y_{i}\right)-1=\sigma_{i} \epsilon,
$$

where the signs $\sigma_{i} \in\{-1,1\}$ must take the values that correspond to this particular vertex. It follows that the solution of (3) can be found by evaluating $\epsilon$ for all systems of the form (4) for different choices for $\sigma_{i}$, and taking the minimum, which boils down to computing the minimum of $\epsilon$ over all supporting systems for $U$, or in other words, computing the elemental thickness of $U$.

The restriction that the family must be parametrized in a linear way may seem to exclude some interesting curves. We will see, however, that at least for ellipses, the family of ellipses can be enlarged to the linear class of axis-aligned conics in such a way that the result of Theorem 1 can be extended to ellipses. 


\subsection{Closed Formula for Algebraic Thickness}

The computation of thickness can be further simplified. We will derive a closed analytical expression for algebraic thickness. To this end we need the following lemma.

Lemma 1. Consider the following system of inequalities in the unknowns $a_{i}$ :

$$
\left|a_{1} g_{1}\left(x_{i}, y_{i}\right)+\cdots+a_{n} g_{n}\left(x_{i}, y_{i}\right)-1\right| \leq \epsilon, \text { for } i=1, \ldots, n+1 .
$$

Let

$$
M=\left(\begin{array}{lll}
g_{1}\left(x_{1}, y_{1}\right) & \ldots g_{n}\left(x_{1}, y_{1}\right) & -1 \\
\ldots & & \\
g_{1}\left(x_{n+1}, y_{n+1}\right) & \ldots g_{n}\left(x_{n+1}, y_{n+1}\right) & -1
\end{array}\right)
$$

denote the coefficient matrix of (5), and let $D_{i}$ denote the cofactor of the element on the last column and $i$-th row of $M$. If at least one of the cofactors is non zero, then (5) has a solution if and only if $\epsilon$ satisfies $\left|\sum_{i} D_{i}\right| / \sum_{i}\left|D_{i}\right| \leq \epsilon$. When all the cofactors are zero then the system has a solution for $\epsilon=0$.

Proof. Assume all the cofactors of the last column are zero. If we then replace the inequalities by equalities and $\epsilon$ by zero in (5), we obtain a homogeneous system. Since all cofactors $D_{i}$ are zero, the determinant of $M$ is zero, and therefore the homogeneous system has a non-trivial solution. Furthermore, this solution is also a solution of the original system (5) with $\epsilon=0$. Next, assume that at least one of the cofactors is non-zero and that (5) has a solution. By multiplying the $i$-th inequality by the cofactor $D_{i}$, and after adding the left and the right sides of the inequalities separately, we find that $\sum_{i}\left|D_{i}\right|\left|a_{1} g_{1}\left(x_{i}, y_{i}\right)+\cdots+a_{n} g_{n}\left(x_{i}, y_{i}\right)-1\right| \leq \epsilon \sum_{i}\left|D_{i}\right|$, and therefore also $\left|\sum_{i} D_{i}\left(a_{1} g_{1}\left(x_{i}, y_{i}\right)+\cdots+a_{n} g_{n}\left(x_{i}, y_{i}\right)-1\right)\right| \leq \epsilon \sum_{i}\left|D_{i}\right|$. However, the terms $\sum_{i} D_{i} g_{j}\left(x_{i}, y_{i}\right)$ vanish for all $j$, because each term represents the determinant of a matrix with two identical columns. Hence, $\left|\sum_{i} D_{i}(-1)\right|=\sum_{i}\left|D_{i}\right| \leq \epsilon \sum_{i}\left|D_{i}\right|$, which concludes the first part of the proof.

The converse is proved by contra position. Assume that (5) does not have a solution. Then for each vector $\left(\delta_{1}, \ldots, \delta_{n+1}\right)$ that satisfies $\left|\delta_{i}\right| \leq \epsilon$, the system of $n+1$ linear equations in $n$ unknowns

$$
a_{1} g_{1}\left(x_{i}, y_{i}\right)+\cdots+a_{n} g_{n}\left(x_{i}, y_{i}\right)-\left(1+\delta_{i}\right)=0, \text { for } i=1, \ldots, n+1
$$

does not have a solution either. Therefore the homogeneous system with $n+1$ equations

$$
a_{1} g_{1}\left(x_{i}, y_{i}\right)+\cdots+a_{n} g_{n}\left(x_{i}, y_{i}\right)-\left(1+\delta_{i}\right) b=0, \text { for } i=1, \ldots, n+1
$$

in the $n+1$ unknowns $a_{1}, \ldots, a_{n}, b$ cannot have a non-trivial solution. Since, if such a solution would exist, it would have the form $\left(a_{1}, \ldots, a_{n}, b\right)$, with $b \neq 0$, because the columns of the matrix $m_{i j}=g_{j}\left(x_{i}, y_{i}\right)$ are independent. Then $\left(a_{1} / b, \ldots, a_{n} / b, 1\right)$ would be a solution of (7), and $\left(a_{1} / b, \ldots, a_{n} / b\right)$ a solution of (6). Since we assume that no such solution exists, it follows that the determinant of the system matrix of (7) cannot vanish, that is, $\left|\sum_{i} D_{i}\left(1+\delta_{i}\right)\right|>0$, for $\delta_{i}$ satisfying $\left|\delta_{i}\right| \leq \epsilon$. Since, $\sum_{i} D_{i} \delta_{i}$ can take any value in the closed interval $\left[\epsilon \sum_{i}\left|D_{i}\right|, \epsilon \sum_{i}\left|D_{i}\right|\right]$, it follows that $\left|\sum_{i} D_{i}\right|>\sum_{i}\left|D_{i}\right|$. 
A closed formula for the algebraic thickness follows easily now.

Theorem 2. Let $C$ be the family of curves defined by $a_{1} g_{1}(x, y)+\cdots+a_{n} g_{n}(x, y)=1$. Let $U$ be an elemental subset of $n+1$ points $\left(x_{i}, y_{i}\right)$, and let $M$ and its cofactors $D_{i}$ be defined as in Lemma 1 If the determinant of $M$ is non-zero, then the algebraic thickness of $U$ is equal to $\left|\sum_{i} D_{i}\right| / \sum_{i}\left|D_{i}\right|$. If the determinant of $M$ is zero, then the algebraic thickness is also zero.

Proof. Reconsider the linear programming problem (3), which corresponds to finding the algebraic thickness. This minimization problem can be reformulated as follows. What is the minimum value for $\epsilon$ such that the polyhedron defined by the constraints in (3) is still non-empty? By Lemma 1 this minimal value for $\epsilon$ is given by $\left|\sum_{i} D_{i}\right| / \sum_{i}\left|D_{i}\right|$, when $\operatorname{det} M \neq 0$.

\subsection{Algebraic Thickness of Non-elemental Sets}

The algebraic thickness of a larger set can be computed from the algebraic thickness of its elemental subsets.

Theorem 3. Let $C$ be the family of curves defined by $a_{1} g_{1}(x, y)+\cdots+a_{n} g_{n}(x, y)=1$. The algebraic thickness of a set $S$ is equal to the maximum of the algebraic thicknesses of all its elemental subsets.

Proof. By definition the algebraic thickness is equal to the minimum value for $\epsilon$ of the following optimization problem:

Minimize the objective function $\epsilon$ subject to the constraints

$$
\left|a_{1} g_{1}\left(x_{i}, y_{i}\right)+\cdots+a_{n} g_{n}\left(x_{i}, y_{i}\right)-1\right| \leq \epsilon, \quad\left(x_{i}, y_{i}\right) \in S \text {. }
$$

Let $\epsilon$ be the minimal value so that we can still find values for $a_{1}, \ldots, a_{n}$ that satisfy the above inequalities. Given $\epsilon$ the inequalities define a convex set in the parameter space $\mathbb{R}^{n}$. According to Helly's theorem this convex set is non-empty provided the intersection of every $n+1$ of its subsets is non-empty [3]. Or in other words, for the constraints to be satisfied, it suffices that they are satisfied for the points $\left(x_{i}, y_{i}\right)$ of each elemental subset $U$ of $S$ separately. It follows that the minimal value for $\epsilon$ is the maximum of $\tau_{a}(U)$, where $U$ ranges over all the elemental subsets of $S$.

\section{Axis-Aligned Ellipses}

We will apply the previous results to conics, and in particular ellipses. An ellipse has an equation of the form

$$
\frac{(x-p)^{2}}{r^{2}}+\frac{(y-q)^{2}}{s^{2}}=1 .
$$

We will denote this family of curves as $C_{\text {ellipse }}$. Since this family is characterized by 4 parameters, each elemental subset $U$ has 5 points, i.e., $U=\left\{\left(x_{1}, y_{1}\right), \ldots,\left(x_{5}, y_{5}\right)\right\}$. In 
$C_{\text {ellipse }}$ a signed elemental subset is part of a supporting system if the following system has a solution for $p, q, r, s$ and $\epsilon_{\text {ellipse: }}$ :

$$
\left\{\begin{array}{l}
\frac{\left(x_{1}-p\right)^{2}}{r^{2}}+\frac{\left(y_{1}-q\right)^{2}}{s^{2}}-1=\sigma_{1} \epsilon_{\text {ellipse }} \\
\cdots \\
\frac{\left(x_{5}-p\right)^{2}}{r^{2}}+\frac{\left(y_{5}-q\right)^{2}}{s^{2}}-1=\sigma_{5} \epsilon_{\text {ellipse }}
\end{array}\right.
$$

Although this system is non-linear it can easily be handled by equation solvers to determine the two supporting curves and the best elemental fit. Within the family of ellipses not every signed elemental subset will give rise to a supporting system, however. The existence of a supporting system can be determined by extending the family of ellipses to the family conics $C_{\text {conic }}$ which consists of curves of the form

$$
a x_{i}^{2}+b y_{i}^{2}+d x_{i}+e y_{i}-1=0 .
$$

This family includes parabola, hyperbola, and ellipses. Since the term $x y$ is missing, all conics are axis-aligned. The family of conics is also characterized by 4 parameters and has therefore elemental subsets with 5 points.

In $C_{\text {conic }}$ a signed elemental subset is part of a supporting system provided the following system can be solved:

$$
\left\{\begin{array}{l}
a x_{1}^{2}+b y_{1}^{2}+d x_{1}+e y_{1}-\sigma_{1} \epsilon_{c o n i c}=1 \\
\cdots \\
a x_{5}^{2}+b y_{5}^{2}+d x_{5}+e y_{5}-\sigma_{5} \epsilon_{c o n i c}=1
\end{array}\right.
$$

Therefore the signed elemental subset is part of a supporting system when the determinant of the following matrix is non-zero:

$$
M_{\text {conic }}=\left(\begin{array}{lllll}
x_{1}^{2} & y_{1}^{2} & x_{1} & y_{1} & \sigma_{1} \\
\cdots & & & \\
x_{5}^{2} & y_{5}^{2} & x_{5} & y_{5} & \sigma_{5}
\end{array}\right)
$$

When a signed elemental subset gives rise to a supporting system in $C_{\text {conic }}$ then the solution we find for $\epsilon_{\text {conic }}$ yields the elemental thickness $\tau_{e}\left(U_{\sigma}, C^{0}, C^{+\epsilon}, C^{-\epsilon}\right)$. The elemental thickness of $U$ itself, was defined as $\tau_{e}(U)=\min _{\sigma} \tau_{e}\left(U_{\sigma}, C^{0}, C^{+\epsilon}, C^{-\epsilon}\right)$ where the minimum is taken over all supporting systems of $U$.

\subsection{Relation between Elliptical and Conic Fits}

By Theorems 1 and 2 the elemental thickness of $U$ in $C_{\text {conic }}$ is equal to

$$
\tau_{e, \text { conic }}(U)=\tau_{a, \text { conic }}(U)=\frac{\left|D_{1}+D_{2}+D_{3}+D_{4}+D_{5}\right|}{\left|D_{1}\right|+\left|D_{2}\right|+\left|D_{3}\right|+\left|D_{4}\right|+\left|D_{5}\right|},
$$

where $D_{i}$ denotes the cofactor of the element on the last column and $i$-th row of $M_{\text {conic }}$.

In general, we have $\tau_{e, \text { ellipse }}(U) \neq \tau_{e, \text { conic }}(U)$. Nevertheless, when they exist, the solutions of (9) and (10) are closely related. A solution of (9) can always be rewritten as a solution for (10). Conversely, suppose we find a solution for (10). Then this conic will 
be an ellipse if the parameters $a$ and $b$ have the same sign, and are both non-vanishing. In that case the subset also has a supporting system in the family of ellipses. Since these parameters are always unique, we have the following result: A signed elemental subset has at most one supporting system within the family of ellipses. We note that this is not true in general. For example, for lemniscates a single signed elemental set may be part of more than one supporting system.

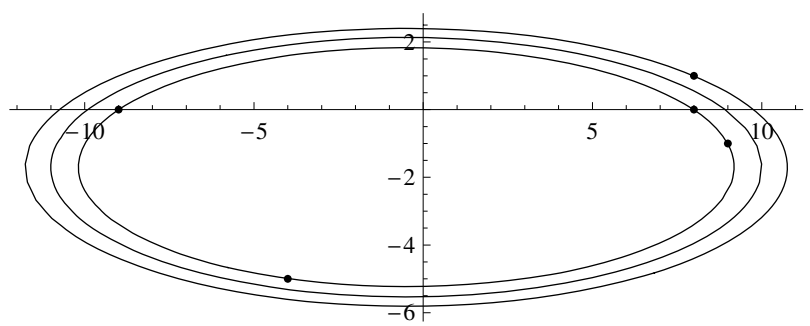

Fig. 3. A supporting system of 5 points in the family of aligned conics

Fig. 3 shows five points of an elemental subset. The best fit and the two supporting curves $C^{+\epsilon}, C^{-\epsilon}$ have been computed using (10) for all 32 possible sign vectors. Fig. 3 shows one particular supporting system where the signs have been attributed so that 4 points lie on the inner ellipse $C^{-\epsilon}$ and one point lies on the outer ellipse $C^{\epsilon}$. The supporting system with the smallest value for $\epsilon_{\text {conic }}$ is the one shown in Fig. 4 For some choices of the signs $\sigma_{i}$ we obtain a hyperbola as best fit in $C_{c o n i c}$, as shown in Fig. 5. This means that when the signs are attributed as in Fig. 5, we will not find a real solution when we use (9) to compute a supporting system in $C_{\text {ellipse }}$.

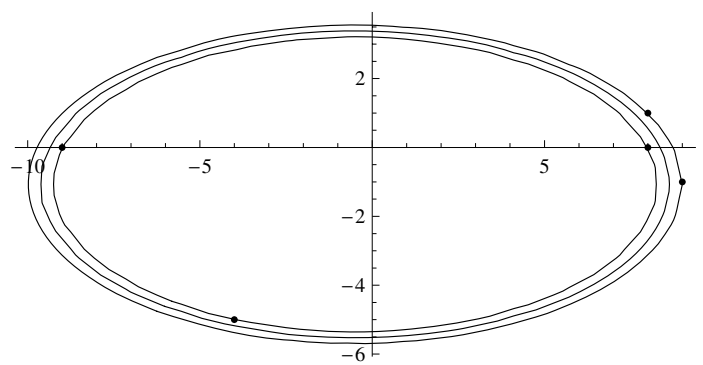

Fig. 4. The supporting system that yields the minimum elemental thickness

The elemental thickness $\tau_{e, \text { conic }}$ of an elemental subset in $C_{\text {conic }}$ is not the same as its elemental thickness $\tau_{e, \text { ellipse }}$ in $C_{\text {ellipse }}$. The supporting curves are the same however. Let

$$
a x^{2}+b y^{2}+d x+e y=1
$$

be the best fit found for a signed elemental subset $U_{\sigma}$ in the family $C_{\text {conic }}$, and let

$$
a x^{2}+b y^{2}+d x+e y=1 \pm \epsilon_{\text {conic }}
$$




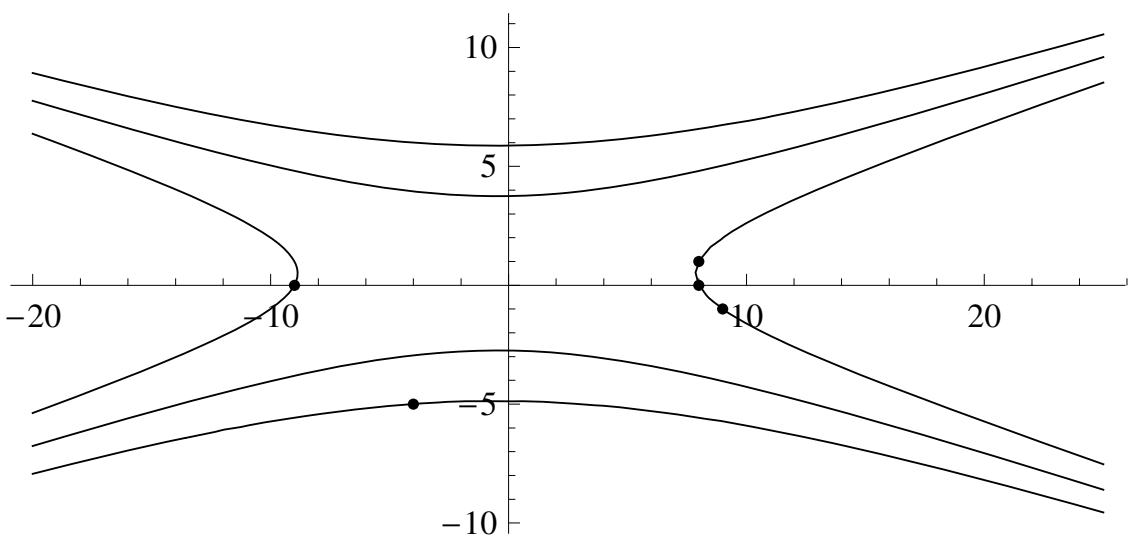

Fig. 5. A supporting system consisting of hyperbola

be its two supporting curves. We can rewrite (13) as $\frac{(x-p)^{2}}{r^{2}}+\frac{(y-q)^{2}}{s^{2}}=1$ and (14) as $\frac{(x-p)^{2}}{r^{2}}+\frac{(y-q)^{2}}{s^{2}}=1 \pm \epsilon_{\text {ellipse }}$ where $p=\frac{-d}{2 a}, q=\frac{-e}{2 b}, r^{2}=\frac{4 a b+b d^{2}+a e^{2}}{4 a^{2} b}, s^{2}=$ $\frac{4 a b+b d^{2}+a e^{2}}{4 a b^{2}}$, and $\epsilon_{\text {ellipse }}=\frac{4 a b \epsilon_{c o n i c}}{4 a b+b d^{2}+a e^{2}}$. The three curves defined by the rewritten expressions clearly yield a supporting system for $U_{\sigma}$ in $C_{\text {ellipse }}$.

\subsection{Real Thickness}

The elemental thickness and the algebraic thickness can be easily calculated, but since they are based on algebraic measures rather than geometrical ones, they differ from the thickness as we would define it using Euclidean distance functions. In particular, the elemental thickness $\tau_{e, \text { conic }}(U)$ is not translation invariant. That is, if we translate all the points of $U$ by a translation vector to obtain a second set $V$, then the thickness of $V$ will be not the same as the thickness of $U$.

On the other hand, the elemental thickness $\tau_{e, \text { ellipse }}(U)$ is translation invariant. In fact, $\tau_{e, e l l i p s e}(U)$ is invariant for any transformation of the form

$$
\left(\begin{array}{l}
x^{\prime} \\
y^{\prime} \\
1
\end{array}\right)=\left(\begin{array}{lll}
\alpha_{11} & 0 & \alpha_{13} \\
0 & \alpha_{22} & \alpha_{13} \\
0 & 0 & 1
\end{array}\right)\left(\begin{array}{l}
x \\
y \\
1
\end{array}\right) .
$$

For example, after scaling by a factor 2 , the thickness $\tau_{e, \text { ellipse }}(U)$ of the transformed set will still be that of the original elemental set, although the transformed points will lie apart twice as far. Hence neither $\tau_{\text {e,ellipse }}(U)$ or $\tau_{e, \text { conic }}(U)$ are good measures for the real distance between the supporting curves of an elemental set. Neither $\tau_{e, c o n i c}(U)$ nor $\tau_{e, \text { ellipse }}(U)$ behave as normal thickness functions, because the first is not invariant for translations, while the second is invariant even for scaling.

A simple calculation shows, however, that the Euclidean distance between the two supporting ellipses can be easily be derived from the two supporting curves. We find

$$
\begin{aligned}
& \tau_{\text {major }}=r\left(\sqrt{1+\tau_{\text {e,ellipse }}}-\sqrt{1-\tau_{e, \text { ellipse }}}\right) \\
& \tau_{\text {minor }}=s\left(\sqrt{1+\tau_{\text {e,ellipse }}}-\sqrt{1-\tau_{e, \text { ellipse }}}\right)
\end{aligned}
$$


as the distance between the two supporting curves when measured along the ellipses major and minor axis, respectively.

\subsection{Fitting Ellipses to Real Data Sets}

For sets of data points that contain more than 5 points, we can apply Theorem 3 Fig. 6 shows the result. The thickness was computed for each elemental subset of a set $S$ of 20 points, using (12). According to Theorem 3 the elemental subset $U$ with the largest thickness must yield a best fit for the entire data set $S$. For this elemental subset $U$ we computed all the supporting with different sign vectors, and selected the supporting system which produced the correct thickness. This supporting system is shown in Fig. 6 The supporting curves enclose the entire set as tightly as possible. The 5 points of the elemental subset $U$ lie on the two supporting ellipses.

Although this approach produces a correct result, its major drawback is that we must compute the thickness of a large collection of elemental subsets (all 5-point subsets of $S$ ).

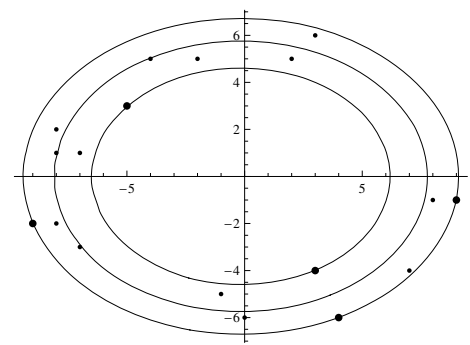

Fig. 6. Best fit and supporting system for non-elemental sets
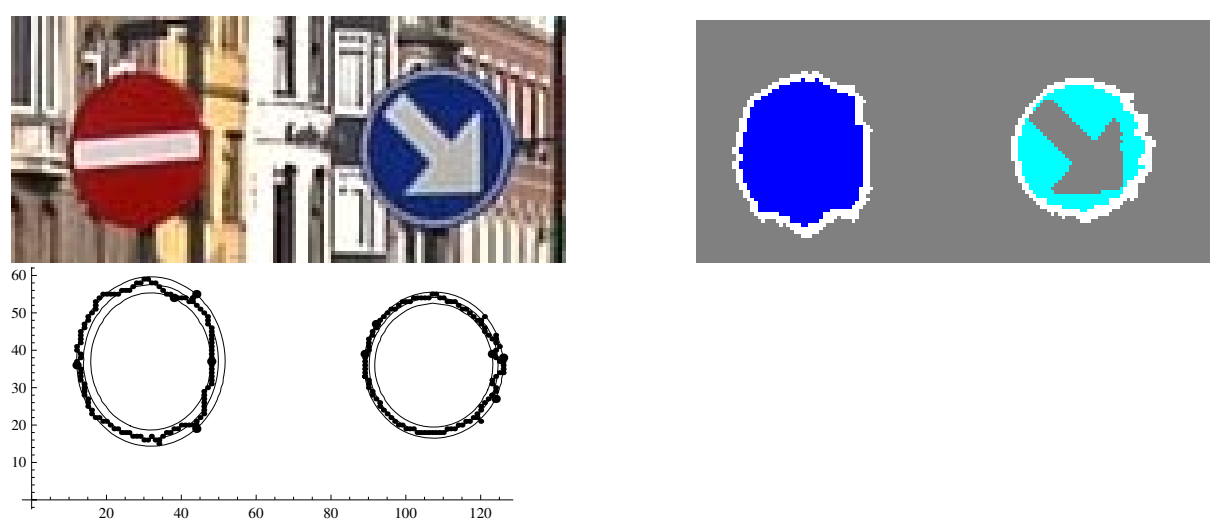

Fig. 7. Ellipse fitting of road sign contours, which were extracted by colour segmentation. The ellipses were fitted by a RANSAC process. The fitting cost was computed for randomly chosen elemental subsets from the edge map, typically between 1000 and 10000 subsets. To be robust against outliers, the RANSAC process stopped when $90 \%$ of the edge points of a closed contour were in the region between $C^{+\epsilon}, C^{-\epsilon}$. 
Fig.7 7 shows a typical application where ellipses were fitted to road sign contours. Computing all elemental thicknesses becomes impractical here because there are too many data points. In [5] it has been shown, however, that a small subcollection of elemental sets is sufficient to obtain a reliable supporting system for parabola. The same technique can be used to estimate a reliable best fit for ellipses. In paricular, only a relatively small number of elemental subsets were used to obtain the fits in Fig. 7 .

\section{Conclusion and Future Work}

We have introduced the two important concepts of elemental and algebraic thickness, a distinction that as far as we know, has not been made before. Although their values coincide for linearly defined families of curves, they are computed in fundamentally different ways. Furthermore, the most interesting question for future research is whether we can find a relation between algebraic and elemental thickness for more generally defined families of implicit curves.

\section{References}

1. Coeurjolly, D., Gerard, Y., Reveilles, J.-P., Tougne, L.: An elementary algorithm for digital arc segmentation. Discrete Applied Mathematics 139, 31-50 (2004)

2. Deboeverie, F., Veelaert, P., Philips, W.: Face recognition using parabola edge map. In: Blanc-Talon, J., Bourennane, S., Philips, W., Popescu, D., Scheunders, P. (eds.) ACIVS 2008. LNCS, vol. 5259, pp. 994-1005. Springer, Heidelberg (2008)

3. Stoer, J., Witzgall, C.: Convexity and Optimization in Finite Dimensions I. Springer, Berlin (1970)

4. Veelaert, P.: Constructive fitting and extraction of geometric primitives. CVGIP: Graphical Models and Image Processing 59, 233-251 (1997)

5. Veelaert, P., Teelen, K.: Fast polynomial segmentation of digitized curves. In: Kuba, A., Nyúl, L.G., Palágyi, K. (eds.) DGCI 2006. LNCS, vol. 4245, pp. 482-493. Springer, Heidelberg (2006)

6. Qiao, Y., Ong, S.H.: Arc-based evaluation and detection of ellipses. Pattern Recognition 40, 1990-2003 (2007)

7. Umbach, D., Jones, K.: A few methods for fitting circles to data. IEEE Trans. on Instrumentation and Measurement 52, 1881-1885 (2003)

8. Zhang, S.-C., Liu, Z.-Q.: A robust, real-time ellipse detector. Pattern Recognition 38, 273-287 (2005)

9. Zunic, J., Sladoje, N.: Efficiency of characterizing ellipses and ellipsoids by discrete moments. IEEE Trans. on Pattern Analysis and Machine Intelligence 22, 407-414 (2000) 